Bulgarian Academy of Sciences. Space Research and Technology Institute. Aerospace Research in Bulgaria. 31, 2019, Sofia

DOI: https://doi.org/10.3897/arb.v31.e11

\title{
APPLYING POTENTIAL-BASED PANEL METHOD FOR STEADY FLOW ANALYSIS ACROSS A WING WITH FINITE SPAN
}

\author{
Konstantin Metodiev \\ Space Research and Technology Institute - Bulgarian Academy of Sciences \\ e-mail: komet@space.bas.bg
}

Keywords: Panel method, Iterative scheme, Finite span wing, Potential flow.

\begin{abstract}
In the paper hereby, an incompressible irrotational steady flow across a submerged body with finite dimensions will be studied. For this purpose, it is necessary to solve Laplace's differential equation about a potential function in order to obtain the conservative velocity vector field. A general solution to the problem utilizing the Green identity implies the double layer potential function at an arbitrary point not belonging to the boundary surface. The potential is expressed by source/sink and doublet singularities distributed over the body surface and a wake attached to the trailing edge. The wake ensures that the Kutta condition is fulfilled. The submerged body geometry is approximated further by quadrilateral panels in order to compute the surface integrals for each panel exactly. To form a linear non-homogenous algebraic system, it is essentially to compute each panel influence to a collocation point of interest. The obtained coefficient matrix is diagonally dominant. The system is solved iteratively by means of the Gauss-Seidel method.

The goal is development of a non-proprietary source code in order to work out a solution to the stated problem. The developed source code is authentic. Auxiliary libraries have not been used. Validation case and numerical results are depicted and discussed in the paper.
\end{abstract}

\section{Introduction}

The proposed approach towards working out a solution to the stated fluid problem utilizes the so-called double layer potential method applied to the Laplace's equation. In this study case, the flow is assumed irrotational and incompressible. This is a relatively old method which has been thoroughly studied and many solution codes have been developed as well. Nevertheless, one advantage of the method provokes development of the current study case: the method is fast and applicable to complex geometries of thick bodies generating lift. What is more, by solving the Laplace's equation the velocity vector field might be found out prior to using equations of motion, such as Euler or Navier-Stokes.

The presented study emphasizes on applicability of iterative schemes for working out a solution to a non-homogenous linear algebraic system relevant to the 
stated fluid problem. In addition, authentic source code development in $\mathrm{C}$ is yet another project goal. To achieve it, Katz and Plotkin's textbook, [1], was extensively used by the author as a guide throughout the presented study case.

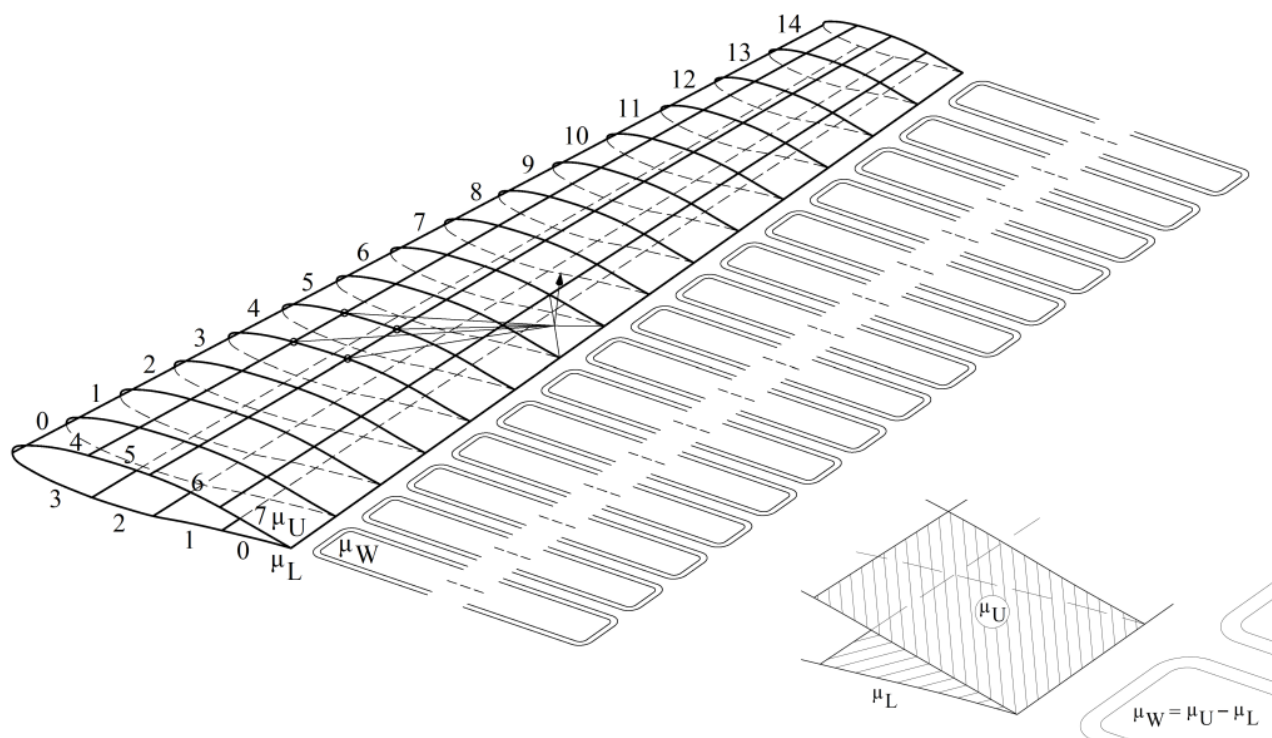

Fig. 1. Wing and a wake shed by the trailing edge. Lower right corner: the wake panel strength computation.

\section{Problem statement and solution}

For an incompressible irrotational flow the continuity equation

(1) $\nabla \mathbf{u}=0$,

takes the form:

$$
\nabla^{2} \Phi=0
$$

where and $\Phi$ is potential function of a conservative velocity field $\mathbf{u}=\operatorname{grad} \Phi$. The boundary condition at surface of a submerged body implies that normal velocity component vanishes:

$$
\nabla \Phi . \mathbf{n}=0 \text {. }
$$

The potential vector grad $\Phi$ is measured in body frame of reference. In addition, a disturbance created by the body decays at infinity $\mathbf{r} \rightarrow \infty$, i.e. 


$$
\lim _{r \rightarrow \infty}\left(\nabla \Phi-\nabla \Phi_{\infty}\right)=0
$$

where $\operatorname{grad} \Phi_{\infty}$ is a vector due to the far field potential. A general solution to the problem stated by formulae $1-4$, might be worked out considering the Green identity. In this way, the potential function at an arbitrary point $\mathrm{P}$ not belonging to the boundary surface is computed by

$$
\Phi(P)=-\frac{1}{4 \pi} \int_{\text {Body }}\left[\sigma\left(\frac{1}{r}\right)-\mu \mathbf{n} . \nabla\left(\frac{1}{r}\right)\right] d S+\frac{1}{4 \pi} \int_{\text {Wake }}\left[\mu \mathbf{n} . \nabla\left(\frac{1}{r}\right)\right] d S+\Phi_{\infty}(P),
$$

where $\sigma$ (source/sink) and $\mu$ (doublet) are flow singularities strengths, $\mathbf{r}$ is distance from point $\mathrm{P}$ to the surface (body, wake, etc.). The surface integrals are taken over the body and a wake model, Fig. 1. The wake is assumed to be thin, so that the dot product $\mathbf{n} \cdot \operatorname{grad} \Phi$ is continuous across it. This means that the wake cannot support fluid-dynamic loads, [1, p. 46]. In order to find the potential function $\Phi$, a unique combination of sources and doublets distribution on the surface must be known in advance, [1, p. 47]. The solution (5) is also denoted as a double layer potential. The integral is computed over a double-sided surface and the normal vector $\mathbf{n}$ points inwards. According to Lamb, [2, p. 40], the impermeability condition (3) results in a constant inner potential

$$
\nabla \Phi . \mathbf{n}=0 \Rightarrow \Phi_{i}=\text { const },
$$

which implies that the current lines are not allowed to enter or leave the inner region - nor they are contained within. If the above equality holds, there can be no fluid motion inside the body. Assuming that the inner potential can be set to $\Phi_{\mathrm{i}}=$ $\Phi_{\infty}=$ const, equation (5) might be rewritten as follows:

$$
-\frac{1}{4 \pi} \int_{\text {Body }}\left[\sigma\left(\frac{1}{r}\right)-\mu \mathbf{n} . \nabla\left(\frac{1}{r}\right)\right] d S+\frac{1}{4 \pi} \int_{\text {Wake }}\left[\mu \mathbf{n} . \nabla\left(\frac{1}{r}\right)\right] d S=0 .
$$

A numerical solution to equation (7) is worked out in the current study.

The boundary conditions (BCs) might either determine the zero normal velocity component (direct, Neumann BCs, also (3)) or specify the velocity potential itself (indirect, Dirichlet BCs) at the boundary surface. Equation (7) interprets the Dirichlet's boundary condition. In addition, it is customary to assign following quantity to the source strength:

$$
\sigma=-\mathbf{n}\left(\mathbf{V}_{\text {linear }}+\boldsymbol{\Omega} \times \mathbf{r}-\mathbf{V}_{\text {free stream }}\right),
$$

where $\mathbf{V}_{\text {linear }}$ is body linear velocity, $\boldsymbol{\Omega}$ is body angular velocity, and $\mathbf{V}_{\text {free stream }}$ is the free stream velocity. 
Further problem refinement is required to describe flow over a thick body with sharp trailing edge generating a lift. In order to hold the rear stagnation point at the trailing edge, sufficient amount of circulation must be created while the body is moving through the fluid. This statement is yet another interpretation of the Kutta condition implying that a jump in the velocity potential exists in the vicinity of the trailing edge and the velocity there is finite.

The wake strength at the trailing edge is determined by setting to zero the vortex element strength located at the trailing edge. Then, the vortex distribution might be regained by an equivalent doublet distribution, [1, p. 250]

$$
\gamma_{T . E .}=-\nabla \mu=0,
$$

which condition is fulfilled if

$$
\mu_{T . E .}=\text { const }=\mu_{W}, \quad \mu_{W}=\mu_{U}-\mu_{L},
$$

where indices W, U, L denote wake, upper, and lower surface respectively, Fig. 1.

The computational algorithm might also be seen in Fig. 1. The influence of singularities distributed onto the body and the wake is computed for each collocation point, which is placed at the panel centroid. In the example depicted in Fig. 1, the collocation point is placed at panel $I=7, j=6$ and the influence panel is $I=5, j=4$. For each collocation, point equation (7) might be written as follows:

$$
\sum_{k=1}^{N} \frac{1}{4 \pi} \int_{B o d y} \mu \mathbf{n} \cdot \nabla\left(\frac{1}{r}\right) d S+\sum_{l=1}^{N_{W}} \frac{1}{4 \pi} \int_{\text {Wake }} \mu \mathbf{n} \cdot \nabla\left(\frac{1}{r}\right) d S-\sum_{k=1}^{N} \frac{1}{4 \pi} \int_{B o d y} \sigma\left(\frac{1}{r}\right) d S=0,
$$

where singularities $\mu$ and $\sigma$ accept unit constant strength. The summation is evaluated for all panels discretizing the body and the wake. To abbreviate the amount of writing further, following symbols are adopted: for a doublet panel

$$
\frac{1}{4 \pi} \int_{\text {Panel }} \mathbf{n} \cdot \nabla\left(\frac{1}{r}\right) d S \equiv C
$$

and for a source element

$$
-\frac{1}{4 \pi} \int_{\text {Panel }}\left(\frac{1}{r}\right) d S \equiv B
$$

The integrals in (12) and (13) solely depend on the panel geometry. Having computed all panel influences onto all collocation points, the following nonhomogenous linear algebraic system is obtained:

$$
\sum_{k=1}^{N} C_{k} \mu_{k}+\sum_{w=1}^{N_{\text {Wake }}} C_{w} \mu_{w}+\sum_{k=1}^{N} B_{k} \sigma_{k}=0
$$


where the source strength $\sigma$ is known at this stage, (8). Equation (14) can be simplified further by computing the wake doublet in terms of the unknown surface doublet, Fig. 1, (also see (10)):

$$
\mu_{W}=\mu_{U}-\mu_{L} .
$$

Then, the influence coefficient takes the form:

$$
C_{W} \mu_{W}=C_{W}\left(\mu_{U}+\mu_{L}\right) .
$$

Therefore, the first two additives in (14) might be grouped which yields the following expression:

$$
\sum_{k=1}^{N} A_{k} \mu_{k}=-\sum_{k=1}^{N} B_{k} \sigma_{k},
$$

where the influence coefficient is computed depending on whether the influence panel is at the trailing edge or not:

$$
\begin{array}{ll}
A_{k}=C_{k} & \text { panel is not at trailing edge } \\
A_{k}=C_{k} \pm C_{w} & \text { panel is at trailing edge }
\end{array}
$$

The expanded form of system (17) is as follows:

$$
\left\|\begin{array}{ccc}
a_{11} & \cdots & a_{1 N} \\
\vdots & \vdots & \vdots \\
a_{N 1} & \cdots & a_{N N}
\end{array}\right\|\left\|\mu_{1}\right\| \begin{gathered}
\vdots \\
\mu_{N}
\end{gathered}\|=-\| \begin{array}{ccc}
b_{11} & \cdots & b_{1 N} \\
\vdots & \vdots & \vdots \\
b_{N 1} & \cdots & b_{N N}
\end{array}\|\| \mid \begin{gathered}
\sigma_{1} \\
\vdots \\
\sigma_{N}
\end{gathered} \|,
$$

where coefficients $a_{i j}$ and $b_{i j}$ are computed according to generic formulae (12) and (13). For particular case of quadrilateral source and doublet with constant strength, formulae derived by Hess and Smith, [3], are used:

- Source

$$
b=-\frac{1}{4 \pi} \sum_{\text {edges }}\left\{\begin{array}{l}
\frac{\left(x-x_{a}\right)\left(y_{b}-y_{a}\right)-\left(y-y_{a}\right)\left(x_{b}-x_{a}\right)}{d_{a b}} \ln \frac{r_{a}+r_{b}+d_{a b}}{r_{a}+r_{b}-d_{a b}}- \\
\left.z \operatorname{atan}\left(\frac{m_{a b} e_{a}-h_{a}}{z r_{a}}\right)-\operatorname{atan}\left(\frac{m_{a b} e_{b}-h_{b}}{z r_{b}}\right)\right]
\end{array}\right\}
$$

- Doublet

$$
a=\frac{1}{4 \pi} \sum_{\text {edges }}\left[\operatorname{atan}\left(\frac{m_{a b} e_{a}-h_{a}}{z r_{a}}\right)-\operatorname{atan}\left(\frac{m_{a b} e_{b}-h_{b}}{z r_{b}}\right)\right] .
$$


In the equation above, following abbreviations are used:

$$
\begin{aligned}
& d_{a b}=\sqrt{\left(x_{b}-x_{a}\right)^{2}+\left(y_{b}-y_{a}\right)^{2}} \quad r_{i}=\sqrt{\left(x-x_{i}\right)^{2}+\left(y-y_{i}\right)^{2}+z^{2}} \\
& m_{a b}=\frac{y_{b}-y_{a}}{x_{b}-x_{a}}, \quad e_{i}=\left(x-x_{i}\right)^{2}+z^{2}, \quad h_{i}=\left(x-x_{i}\right)\left(y-y_{i}\right) \\
& i=a, b
\end{aligned}
$$

where $\mathrm{x}, \mathrm{y}$, and $\mathrm{z}$ are collocation point coordinates and $\mathrm{x}_{\mathrm{a}}, \mathrm{y}_{\mathrm{a}}, \mathrm{z}_{\mathrm{a}}, \mathrm{x}_{\mathrm{b}}, \mathrm{y}_{\mathrm{b}}$, and $\mathrm{z}_{\mathrm{b}}$ are panel corner points coordinates, indices $\mathrm{a}$ and $\mathrm{b}$ denote panel corner points belonging to a same panel edge. All quantities are computed in local (panel) coordinate system, Fig. 3. Hence, preliminary coordinate transformations must be made.

After computing the influence coefficients, a non-homogenous linear algebraic system is obtained in terms of doublet $\mu$ distribution on the wing surface. The system is said to be strictly diagonal dominant if following requirement is met:

$$
\left|a_{i i}\right|>\sum_{\substack{j=1 \\ j \neq i}}^{n}\left|a_{i j}\right|
$$

In other words, the absolute value of each main diagonal element must be greater than sum of absolute values of the remaining elements in the current row respectively. method

If the requirement (23) is met, then the following stationary iterative

$$
x_{i}^{k}=\frac{1}{a_{i i}}\left(b_{i i}-\sum_{j=1}^{i-1} a_{i j} x_{j}^{k}-\sum_{j=i+1}^{n} a_{i j} x_{j}^{k-1}\right) \quad i=1,2, \ldots, n \quad k=1,2,3, \ldots
$$

for solving system (17) is said to converge unconditionally. Method (24) is named after Gauss and Seidel. The formula (24) is a modification of the widely known Jacobi method:

$$
x_{i}^{k}=\frac{1}{a_{i i}}\left(b_{i i}-\sum_{\substack{j=1 \\ j \neq i}}^{n} a_{i j} x_{j}^{k-1}\right) \quad i=1,2, \ldots, n \quad k=1,2,3, \ldots
$$

The convergence criterion used in the algorithm is the relative difference 
Both iterative schemes (24) and (25) require initial guess for the vector $\mathbf{x}$.

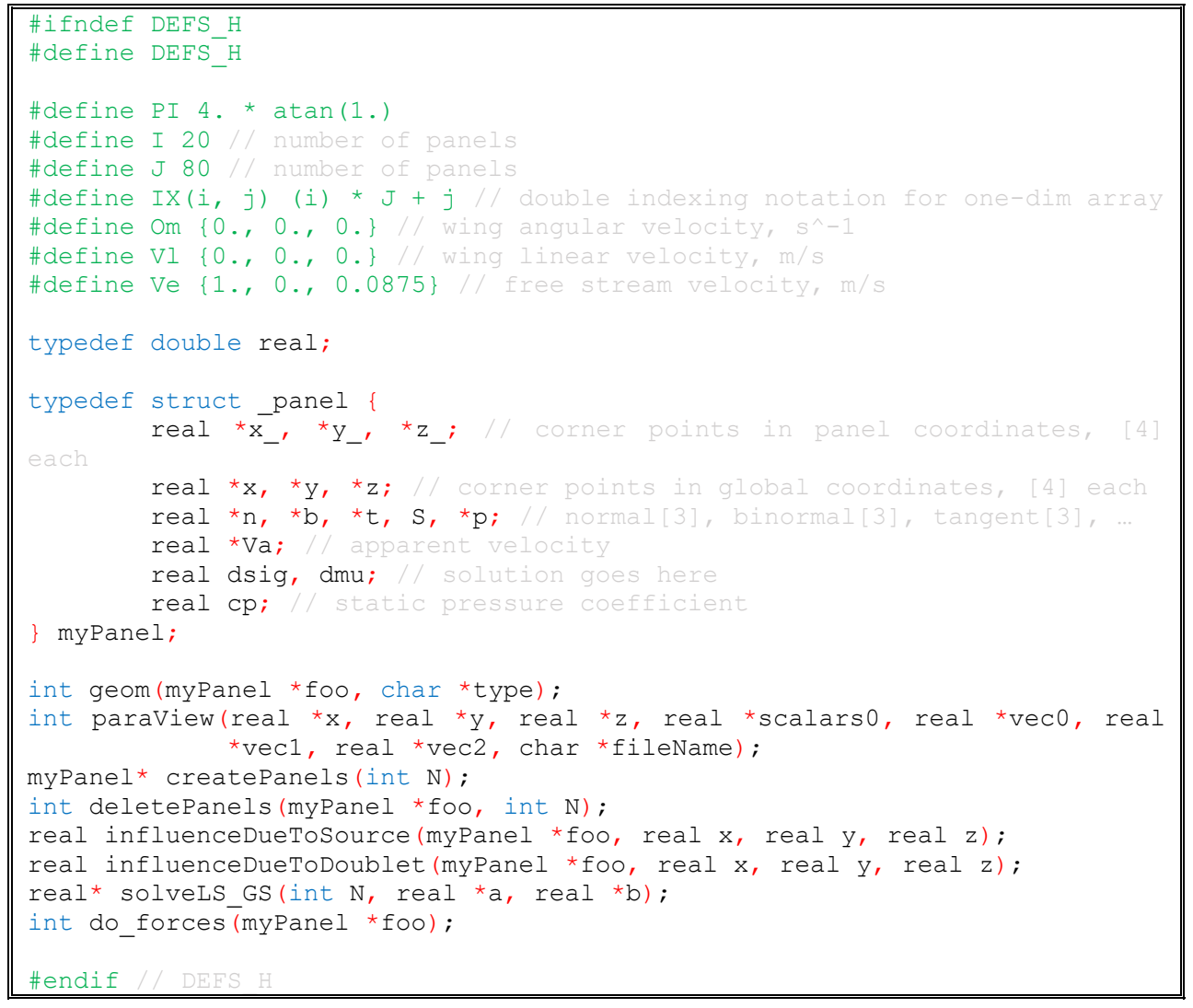

Fig. 2. Source code header file

In Fig. 2, the source header file is shown. Each panel is represented by a structure _panel containing panel geometry, apparent velocity (8), and a few solution quantities, namely singularities strengths and static pressure coefficient. The panel geometry includes corner points coordinates expressed in both global and local (panel) coordinate system. The latter is formed by normal, tangent, and binormal unit vectors as it are shown in Fig. 3 in case of circular cylinder. In addition, the header contains following function prototypes. Function "createPanels" allocates memory for specified number of panels and pointers inside the structure. It returns a pointer to first panel inside the so formed one-dimensional array of structures. Function "deletePanels" does the opposite. Function "geom" 
calculates all necessary geometric parameters relevant to each panel. The remaining function prototypes are self-explanatory and easily understandable. The functions "influenceDueToSource" and "influenceDueToDoublet" compute influences due to singularities at an arbitrary collocation point. The function "solve_GS" solves a linear algebraic system (17) iteratively. It returns a pointer to a solution vector allocated within the function body. What does function "paraView" is arranging the results to meet the "vtk" file requirements [4] and storing them onto the hard drive so that the user can visualize the results by means of a third-party viewer. One dimensional arrays are solely used in the code and accessed by a two-dimensional macro IX(i,j), Fig. 2.

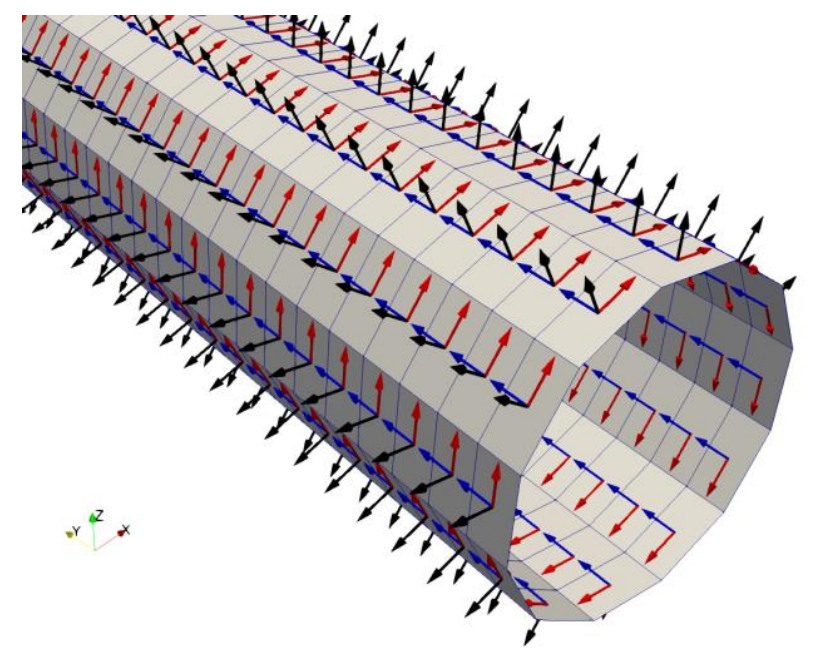

Fig. 3. Local coordinate system for each panel, black-normal, red-tangent, and blue - binormal stored at panel's centroid

\section{Results}

A circular cylinder has been used to validate the developed source code. The static pressure coefficient distribution is visible in Fig. 4. Both front and rear stagnation areas are clearly visible. 


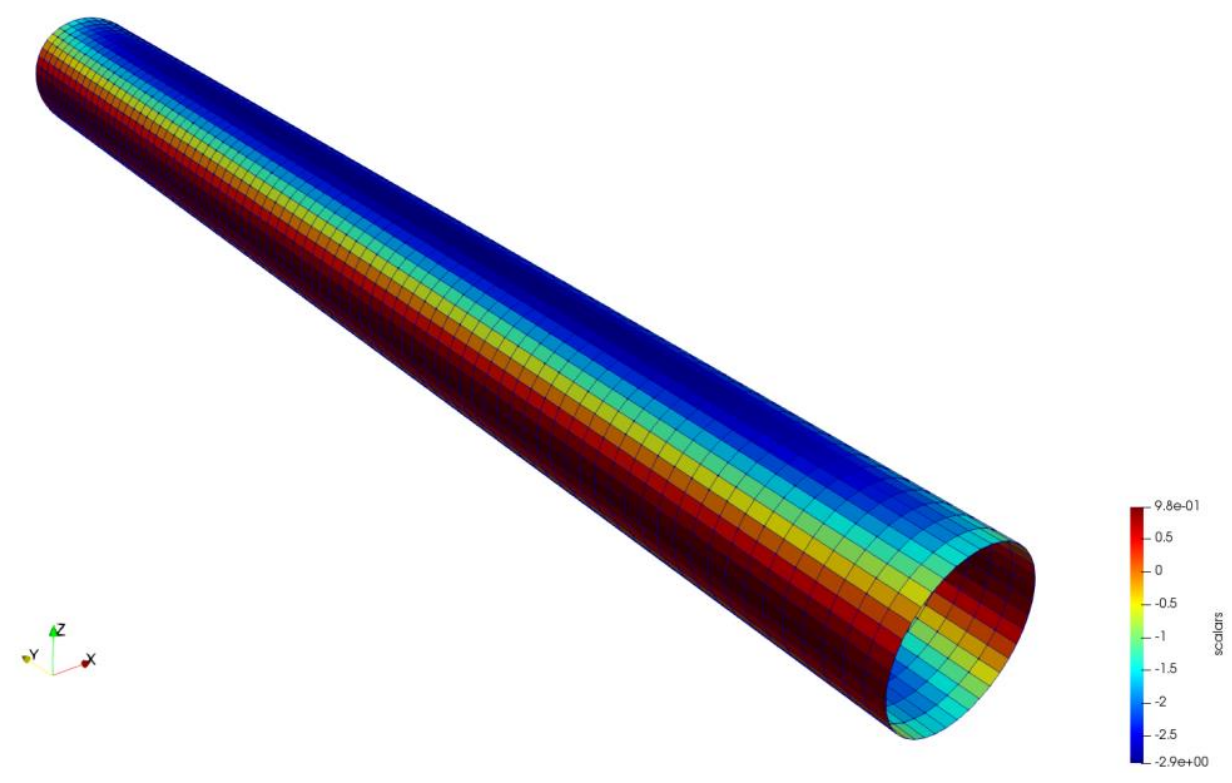

Fig. 4. Static pressure coefficient distribution over a cylinder, $\alpha=0$ deg, $40 \times 80$ panels

\begin{tabular}{|c|c|c|c|c|c|c|c|c|c|c|c|c|}
\hline-0.498 & & & & & & 0.054 & -0.001 & -0.000 & -0.000 & -0.000 & -0.000 & -0.039 \\
\hline 0.000 & 0 & 0.001 & & & $\theta \theta 0$ & 0.001 & 0.054 & & & -0.000 & -0.000 & -0.002 \\
\hline & & & & & & & & & & & & 00 \\
\hline .000 & & 0.000 & 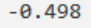 & & 100 & 00 & & & & 901 & 800 & $\theta 00$ \\
\hline & & & & & 01 & 00 & & & & 954 & & $\theta \theta 0$ \\
\hline$\theta$ & & & & & 498 & $\theta 0$ & & & & 01 & 54 & 900 \\
\hline-0.051 & & & & & & $\theta 0$ & & & & 00 & & 954 \\
\hline$-\theta . \epsilon$ & & & & & & $\theta 0$ & $\theta \theta$ & & $\theta \theta$ & 00 & 900 & $\partial 01$ \\
\hline & & & & & & 90 & & & & $\theta \theta$ & $\theta \theta$ & 00 \\
\hline & & & 951 & & & & & & & $\theta 0$ & & 300 \\
\hline & & & & & & & & & & $\theta 0$ & $\theta 0$ & 200 \\
\hline$\theta$. & & & $\theta$ & & -6 & 36 & & & 20 & $\theta 0$ & $\theta \theta$ & 900 \\
\hline-0.036 & & & & & & & & & & $\theta 0$ & & 500 \\
\hline$-\theta$. & & & & & & & & & & $\theta 0$ & & 300 \\
\hline$-\theta$ & & 036 & $\theta 00$ & 0 . & & & & & $\theta 1$ & 00 & $\theta 0$ & 900 \\
\hline & & & $-\theta$ & & & & & & & $\theta 1$ & $\theta 0$ & 900 \\
\hline & & -0.000 & -0.002 & & -0.000 & & & & & 54 & & 200 \\
\hline & & & -0.000 & & -0.036 & & & & $\theta 0$ & 01 & & 200 \\
\hline$-\theta$ & & $\theta 00$ & & & & & & & & 100 & $\theta 0$ & 954 \\
\hline-0.004 & -0 & -0.002 & -0.000 & 0.000 & & & & & & $-\theta$ & 00 & $\partial 01$ \\
\hline-0.000 & & -0.032 & -0.002 & -0.000 & & & & & & $-\theta$ & 00 & $\theta \theta 0$ \\
\hline & & & & & & & & & & & & \\
\hline 0.000 & -0.000 & -0.000 & -0.004 & -0.032 & -0.002 & $-\theta$. & -0.000 & $-\theta$ & -0.002 & -0.039 & -0.002 & -0.000 \\
\hline
\end{tabular}

Fig. 5. Matrix A (eq. 17) upper left corner

Serial computations were also made by means of a finite span wing with airfoil NACA2412 at different angles of attack. Static pressure coefficient distribution is shown in Fig. 6 and Fig. 7. 


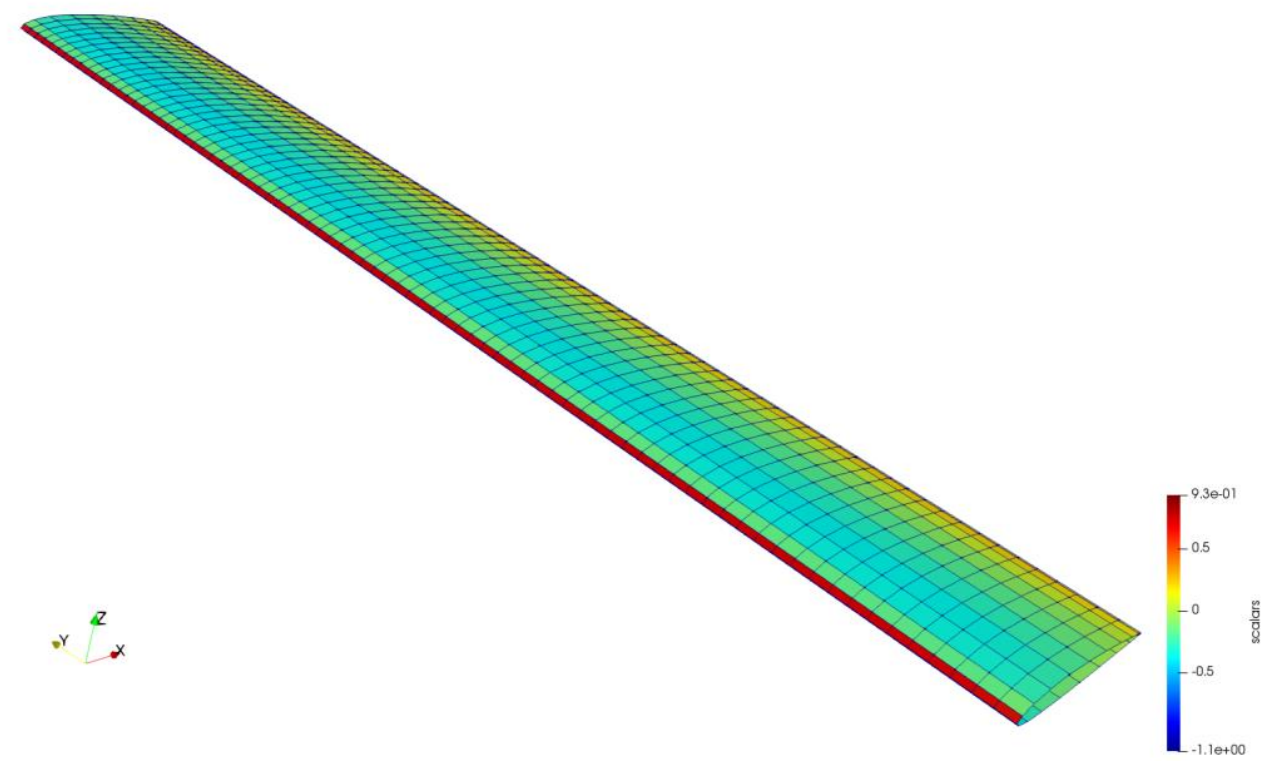

Fig. 6. Static pressure coefficient distribution over a NACA2412 wing, $\alpha=0$ deg, $20 \times 80$ panels

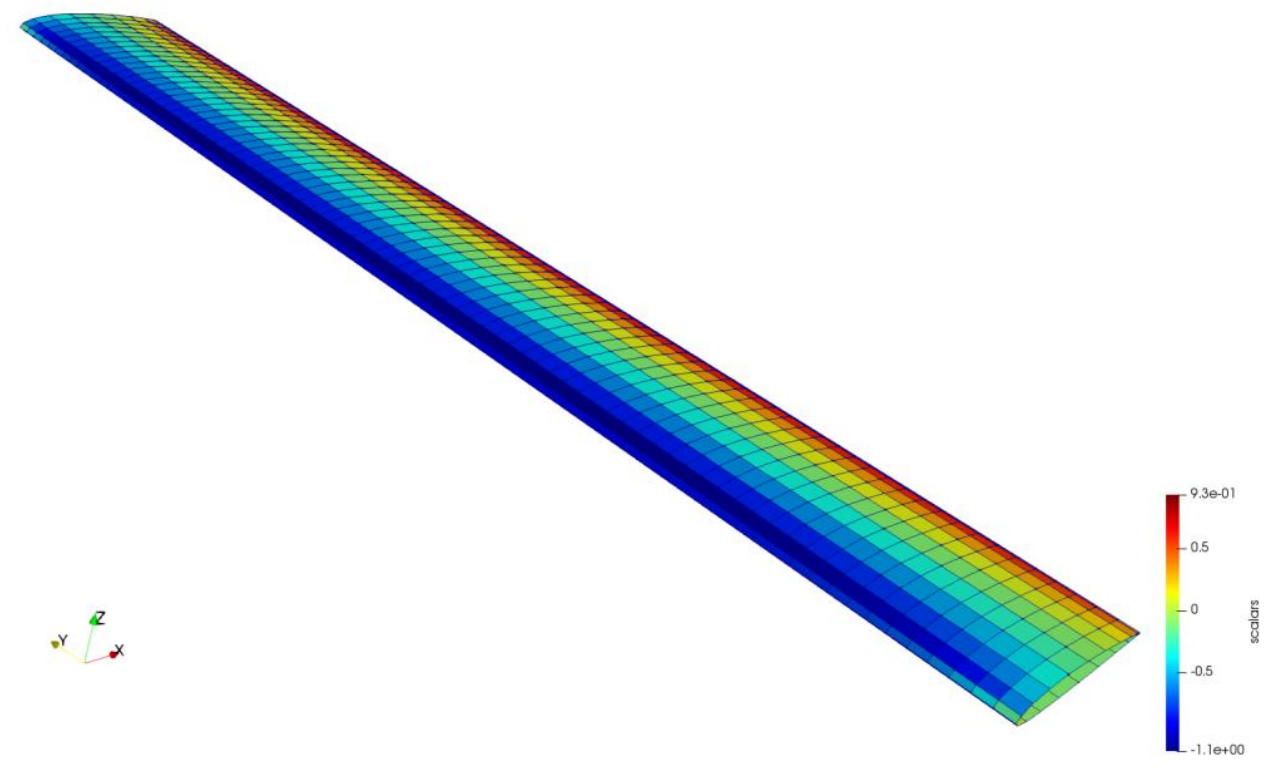

Fig. 7. Static pressure coefficient distribution over a NACA2412 wing, $\alpha=10$ deg, $20 \times 80$ panels 


\section{Discussion}

The obtained static pressure coefficient distribution over a circular cylinder surface fully agrees with what Katz and Plotkin, [1, p. 69], discuss in their textbook. The static pressure coefficient varies within 1 and -3 , so does the same quantity which is shown in Fig. 4. In three-dimensional case, the suction pressures are much smaller, i.e. the so-called "relieving effect" is obtained numerically.

The static pressure coefficient "suction" values are easily observable when the angle of attack is high, Fig. 7, blue region right after the leading edge. The coefficient distribution is symmetric in relation to the mid cross section because the sideslip angle is zero. Although side wing patches are absent, the static pressure coefficient distribution flattens at both wing ends. This result might be explained by the wingtip vortex phenomena.

In Fig. 5, the upper left corner of coefficient matrix A (17) is shown in case of circular cylinder serial computations. The dominating main diagonal could also be noticed. It justifies usage of the iterative scheme (24).

Secondary quantities might be computed further such as lift coefficient and induced drag. Additional demonstration of the Gauss-Seidel method rate of convergence might be seen in [5].

The source code used in the current study is developed by means of Minimalist GNU v. 5.1.0 for Windows. The visualizer used is ParaView v. 5.6.0.

\section{References}

1. Katz, J., A. Plotkin, Low Speed Aerodynamics, $2^{\text {nd }}$ edition, Cambridge University Press, 2001.

2. Lamb, H., Hydrodynamics, $4^{\text {th }}$ edition, Cambridge University Press, 1916.

3. Hess, J. L. and A. M. O. Smith, Calculation of Potential Flow About Arbitrary Bodies, Progress in Aeronautical Sciences, 8, 1967, pp. 1138

4. ASCII VTK Files. https://www.visitusers.org/index.php?title=ASCII_VTK_Files

5. Solution of Linear Equations by Stationary Iterative Methods, 2018.

URL: http://www.space.bas.bg/acsu/Ax_b/ 


\section{ПРИЛОЖЕНИЕ НА ПАНЕЛЕН МЕТОД ЗА АНАЛИЗ \\ НА СТАЦИОНАРНО ТЕЧЕНИЕ ОКОЛО КРИЛО \\ С КРАЙНА РАЗПЕРЕНОСТ}

\section{К. Методиев}

\section{Резюме}

В настоящата статия се изследва течение на несвиваем безвихров поток около тяло с крайни размери. За да се намери консервативния вектор на полето е необходимо да се реши диференциалното уравнение на Лаплас относно потенциална функция. Нетривиално решение на задачата с използване на втора формула на Грин дава като резултат стойност на потенциалната функция на двойния слой в произволна точка от полето, която не принадлежи на граничната повьрхност. Потенциалната функция зависи от особености в полето на течението „източник/падина“ и „дипол“, разпределени по повърхността на тялото, както и от следа, прикрепена към задния ръб на крилото. Следата гарантира удовлетворяването на условието на Кута. Геометрията на тялото се апроксимира с квадратични панели, с цел да се пресметнат лицевите интеграли за всеки панел точно. За да се сведе задачата до линейна алгебрична система е необходимо да се пресметне влиянието на всеки панел в точка от полето. Получената матрица коефициенти е с преобладаващ главен диагонал. Системата се решава итеративно по метода на ГаусЗайдел.

Целта е разработване на сорс код за решаване на поставената задача. Кодът е автентичен и в него не са използвани спомагателни библиотеки. Тестовете за валидация на кода, както и числените резултати са показани графично и обсъдени в статията. 